\title{
THE SHASHLIK ELECTRO-MAGNETIC CALORIMETER FOR THE LHCb EXPERIMENT
}

\author{
S. BARSUK * \\ European Center for Nuclear Research (CERN), \\ Geneva 23, CH-1211, Switzerland \\ and \\ Institute for Theoretical and Experimental Physics (ITEP), \\ B. Cheremushkinskaya 25, Moscow 117218, Russia
}

Representing the $\mathrm{LHCb}$ Calorimeter group

The Shashlik technology has been chosen for the electro-magnetic calorimeter of the LHCb experiment. The detector comprises about 6000 detector cells. This paper summarizes the detector design and production. Also reported are selected performance studies and the detector cells pre-calibration strategy.

Shashlik calorimeter technology, implying a sampling scintillator/lead structure read out by plastic WLS fibers, has been chosen for electromagnetic calorimeter in the $\mathrm{LHCb}$ experiment ${ }^{1}$. This decision was made taking into account good energy resolution, fast time response, acceptable radiation resistance and reliability of the Shashlik technology, as well as the experience accumulated by other experiments ${ }^{2,3,4}$.

The electro-magnetic calorimeter (ECAL) is part of the LHCb calorimeter system ${ }^{5}$, which also comprises the scintillator pad detector, preshower detector and the hadronic calorimeter. The calorimeter will be placed at about $12.5 \mathrm{~m}$ from the interaction point.

The LHCb ECAL provides efficient $\pi^{0}$ and $\eta$ reconstruction in a wide range of momentum, discrimination between electrons, and charged hadrons with overlapping photons, the reconstruction of electrons and high energy $\gamma^{\text {'s }}{ }^{6}$; it also provides a signal to initiate the trigger operation ${ }^{7}$.

The outer dimensions of ECAL match projectively those of the tracking system, $\theta_{x}<300 \mathrm{mrad}$ and $\theta_{y}<250 \mathrm{mrad}$; the acceptance is limited by

*E-mail: serezha.barsuk@cern.ch 
$\theta_{x, y}>25 \mathrm{mrad}$ around the beam pipe due to the substantial radiation dose level. The hit density is a steep function of the distance from the beam pipe, and varies over the active calorimeter surface by two orders of magnitude. The calorimeter is subdivided into the inner, middle and outer sections (Table 1), so that the cell size varies accordingly (Figure 1, left).

Table 1. Main parameters of the LHCb electro-magnetic calorimeter

\begin{tabular}{|l|c|c|c|}
\hline & Inner section & Middle section & Outer section \\
\hline Inner size, $x \times y, \mathrm{~cm}^{2}$ & $65 \times 65$ & $194 \times 145$ & $388 \times 242$ \\
\hline Outer size, $x \times y, \mathrm{~cm}^{2}$ & $194 \times 145$ & $388 \times 242$ & $776 \times 630$ \\
\hline Cell size, $\mathrm{cm}^{2}$ & $4.04 \times 4.04$ & $6.06 \times 6.06$ & $12.12 \times 12.12$ \\
\hline \# of modules & 176 & 448 & 2688 \\
\hline \# of channels & 1472 & 1792 & 2688 \\
\hline \# of cells per module & 9 & 4 & 1 \\
\hline \# of fibers per module & 144 & 144 & 64 \\
\hline Fiber density, $\mathrm{cm}^{-2}$ & 0.98 & 0.98 & 0.44 \\
\hline
\end{tabular}

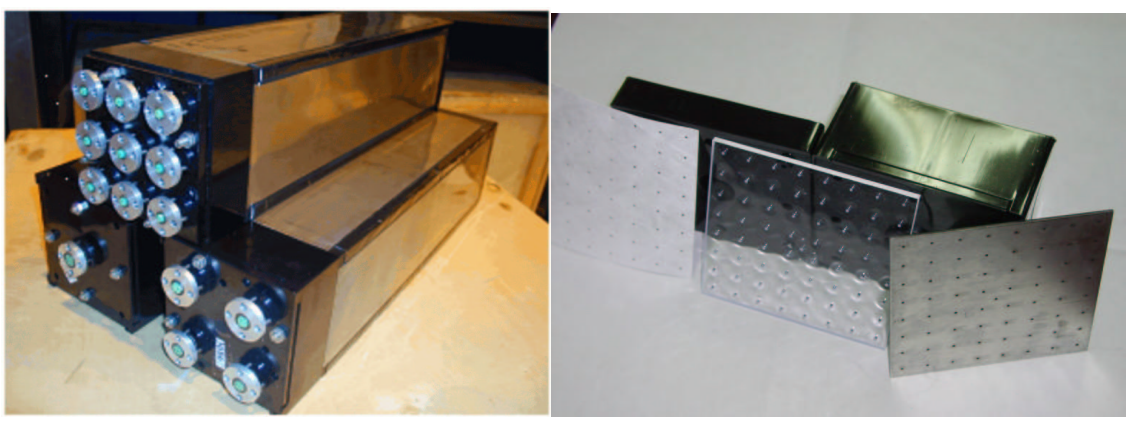

Figure 1. Left: Outer, middle and inner type modules; Right: Scintillator tile, lead plate and TYVEK paper compounds of the stack. Also shown are the housings for fiber loops on the front of the module and fiber bundles on the rear of the module.

The design energy resolution of $\sigma_{E} / E=10 \% / \sqrt{E} \oplus 1 \%(E$ in $G e V$ ) provides $e / h$ separation of better than 100 , and results in a $B$ mass resolution of $65 \mathrm{MeV} / \mathrm{c}^{2}$ for the $B \rightarrow K^{*} \gamma$ penguin decay with a high- $E_{T}$ photon and of $75 \mathrm{MeV} / \mathrm{c}^{2}$ for $B \rightarrow \rho \pi$ decay where the $\pi^{0}$ reconstruction is important.

Module stack is built from alternating layers of $2 \mathrm{~mm}$ thick lead, white reflecting $120 \mu \mathrm{m}$ thick TYVEK paper and $4 \mathrm{~mm}$ thick scintillator tiles (Figure 1, right). In depth, the $66 \mathrm{~Pb}$ and scintillator layers form a $42 \mathrm{~cm}$ stack corresponding to $25 X_{0}$. The Moliere radius of the stack is $3.5 \mathrm{~cm}$.

The stack is wrapped with black paper to ensure light tightness, pressed and fixed from the sides by the welding of $100 \mu \mathrm{m}$ steel tape. 
Scintillator tiles are produced from polystyrene with $2.5 \%$ p-terphenyl and $0.01 \%$ POPOP admixtures. The scintillator tile production employs the high pressure injection molding technique. Tile edges are chemically treated (Figure 2, left) thus providing diffusive reflection in order to improve

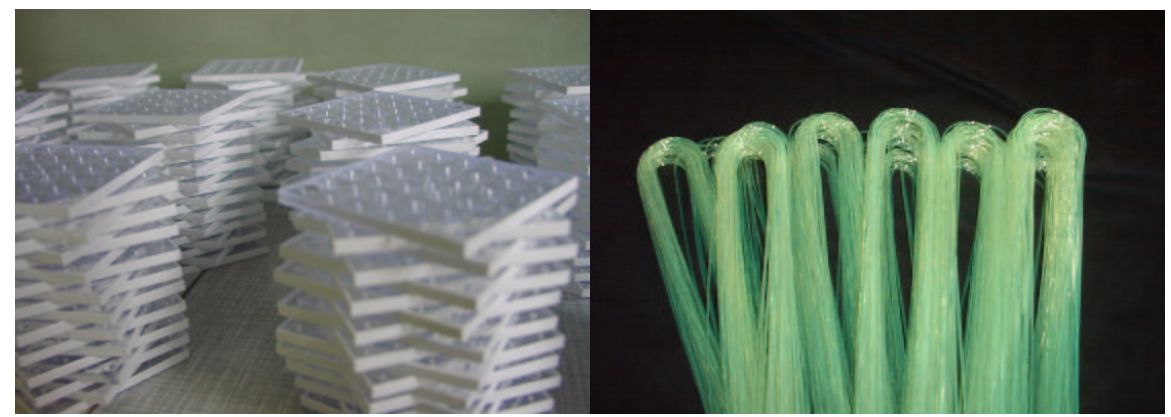

Figure 2. Scintillator tiles with treated edges (left) and WLS fibers with loops corresponding to the front module sides (right)

light collection efficiency, lateral uniformity and prevent tile-to-tile light cross-talk. For the produced tiles the tile to tile light yield fluctuation has an R.M.S. smaller than $2.5 \%$.

The $P b$ plates are produced using sheet-metal stamping.

The light from the scintillator tiles, is re-emitted and transported by $1.2 \mathrm{~mm}$ diameter WLS Y-11(250)MSJ fibers from Kuraray, penetrating the entire module. The fibers belonging to each calorimeter cell are bundled at the end of the module and polished. In order to improve light collection efficiency and lateral uniformity of response, the fibers form loops (Figure 2, right) at the front side of the module. Fiber bending under uniformly distributed dry heat made it possible to produce fiber loops with radii as small as $10 \mathrm{~mm}$, where the light loss is driven mainly by the geometrical optics reflection down the loop. When measured after the fiber loop, the light yield varies from loop to loop with an R.M.S. of the spread of $1.6 \%$.

The light is read out with Hamamatsu R7899-20 phototubes with the high voltage provided by a Cockcroft-Walton base (Figure 3 ).

Lateral non-uniformity in the light collection efficiency comes predominantly from two sources: non-perfect light reflection from tile edges (the so-called global non-uniformity effect), and shower position with respect to the fibers (local or inter-fiber non-uniformity). Global non-uniformity depends on the mean light path, which is a function of tile transparency, 


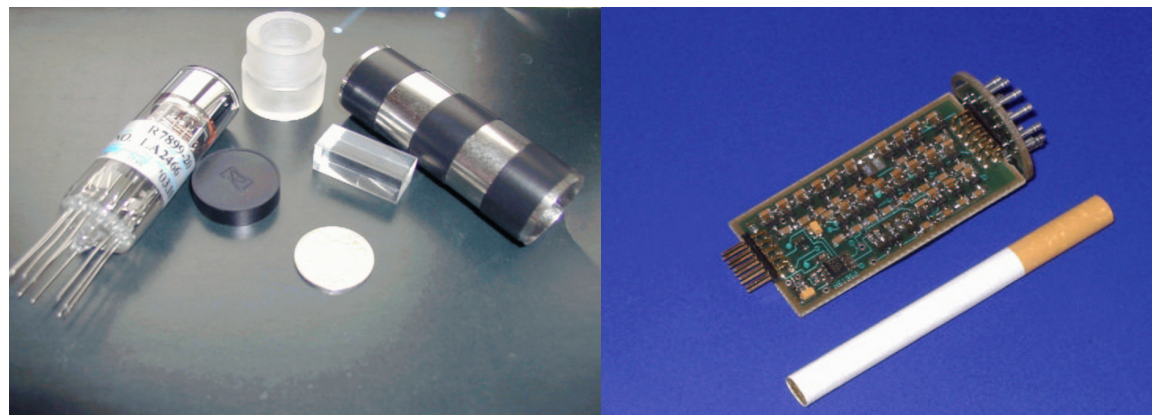

Figure 3. Hamamatsu R7899-20 phototube together with the light mixer, mixer housing and permalloy magnetic screen (left) and the Cockcroft-Walton multiplier (right)

edge reflection quality and fiber density. Local non-uniformity is affected by the inter-fiber distance and diameter of the fiber. Following reference ${ }^{8}$ the non-uniformity effect is parameterized with

$$
f(x) \sim\left[1-A_{g l o b} \cdot\left(\frac{x-x_{0}}{l_{0} / 2}\right)^{2}\right] \times\left[1-A_{l o c} \cdot \cos \left\{\frac{2 \pi}{d} \cdot\left(x-x_{0}\right)\right\}\right],
$$

where $x_{0}$ is a cell center position, $l_{0}$ - cell size, $d$ - inter-fiber distance. The values of $A_{\text {glob }}$ and $A_{l o c}$ determine the size of global and local nonuniformity effect respectfully. Since a new method of tile edge treatment is used, we show the lateral uniformity study. Figure 4 shows the lateral scan of the outer type module with the $50 \mathrm{GeV}$ electron beam. The fit with (1) gives a global uniformity coefficient of $0.5 \%$, and a local uniformity parameter of $0.4 \%$. Significant variations are seen at $\pm 60 \mathrm{~mm}$ from the plot center, corresponding to the transition between the modules. The module response varies within $\pm 1.3 \%$ over the module surface. For realistic incident angles (bigger than $200 \mathrm{mrad}$ ) the response varies by less than $\pm 0.6 \%$ over the module surface. Results on the lateral variation of response are in agreement with the Monte-Carlo simulation. For the inner and middle type modules the $A_{l o c}$ parameter is smaller due to higher fiber density.

The outer module energy resolution (Figure 5) was found to be $\sigma_{E} / E=$ $9.4 \% / \sqrt{E} \oplus 0.8 \% \oplus 0.15 G e V / E$ ( $E$ in $G e V)$. The noise term obtained from the fit, is in agreement with the measurements of pedestal widths. For a few energies we tried to disentangle the contribution from lateral nonuniformity, by comparing the energy resolution obtained in three different geometries: a rectangle (full non-uniformity effect included), a strip (nonuniformity along one coordinate is eliminated) and a spot by averaging the 


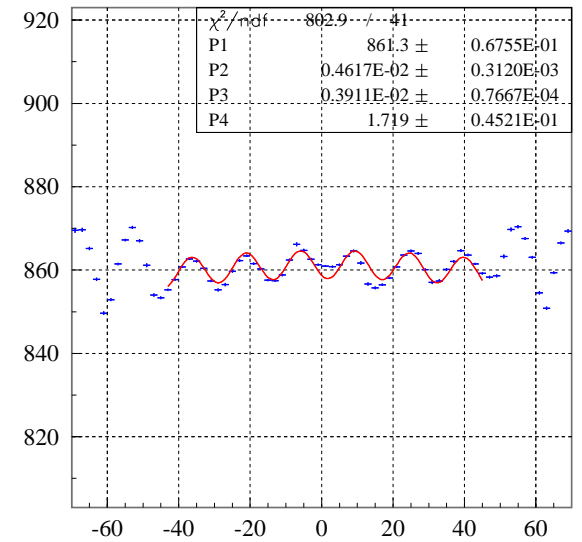

Figure 4. The lateral uniformity of the module response as measured with the $50 \mathrm{GeV}$ electron beam. Note the suppressed zero

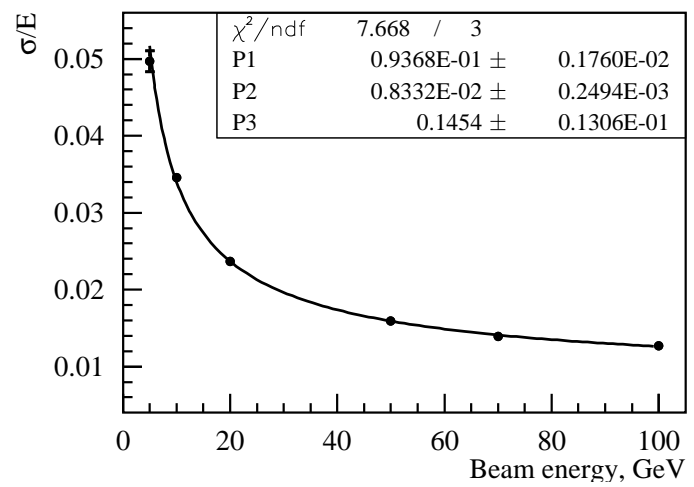

Figure 5. The energy resolution curve of the outer type ECAL module measured with the electron beam

energy resolution curves for four symmetrical $\pm 1 \mathrm{~mm} \times \pm 1 \mathrm{~mm}$ areas across the module surface (Figure 6). The measured constant term in the three geometries was different and its variation was consistent with corresponding lateral non-uniformities measured by the $A_{l o c}$ parameter.

As a final stage of module quality control and as a tool of pre-calibration, the module response to cosmic particles will be measured. The modules are positioned vertically between two planes of scintillators for triggering. Ver- 

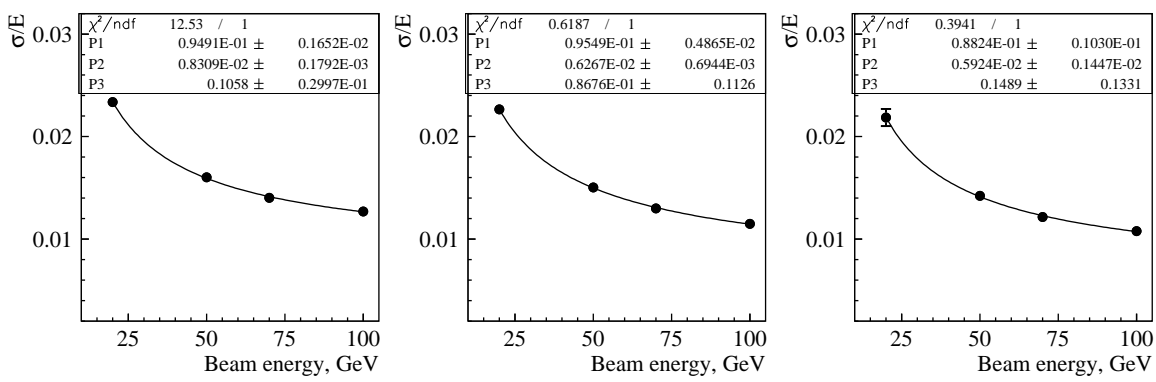

Figure 6. The energy resolution curves for different lateral uniformity contributions: rectangle $(\Delta x, \Delta y)=( \pm 15 m m, \pm 30 m m))$, strip $(\Delta x, \Delta y)=( \pm 1 m m, \pm 30 m m))$ and spot $(\Delta x, \Delta y)=( \pm 1 m m, \pm 1 m m))$

tical tracks contained in a cell are selected by means of veto from neighbor cells, thus sharpening the pulse height distribution at the price of lower trigger rate. Time needed to determine peak position to a precision of about $1 \%$, varies from about 1 hour for outer type cells to about 30 hours for cells of inner type. Figure 7 shows an example of inner type cell re-

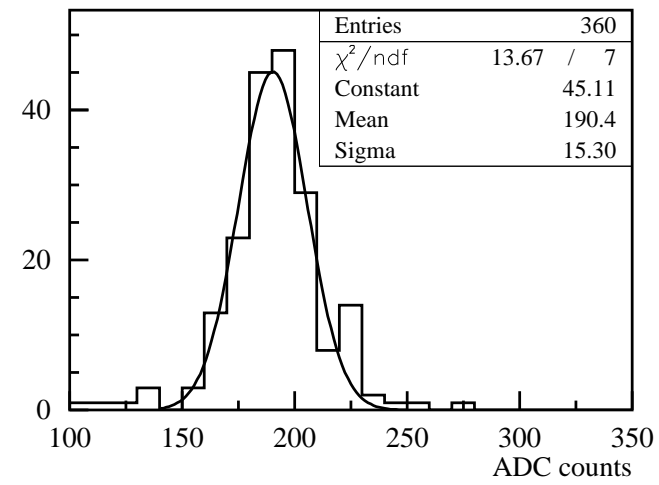

Figure 7. The response of an inner type cell to cosmic particles

sponse to cosmic particles. The stability of the system during the runs will be monitored with pulses from an LED illuminating each photomultiplier. The stability of the LED itself will be monitored with a pin diod.

All the modules have been produced and delivered to CERN. The start of the detector installation is scheduled for fall 2004. 


\section{Acknowledgments}

The results presented are due to collaborative work of many people from the LHCb Calorimeter group, and it is my pleasure to acknowledge their important contribution. I thank V.Rusinov and E.Tarkovsky for valuable discussions and R.Kristic and T.Kvaratskheliia for their help in preparing the talk. I am highly indebted to L.L.Camilleri, A.Golutvin, J.Lefrançois and A.Schopper for carefull reading the manuscript, and many useful comments and suggestions. The detector studies with the e-beam were only possible due to excellent machine operation by the SPS crew at CERN.

\section{References}

1. LHCb Technical Proposal, CERN LHCC 98-4 LHCC/P4, (1998).

2. E.Tarkovsky, Nucl. Instrum. Meth. A379, 515 (1996).

3. A.Bazilevsky et al, IEEE Transactions on Nuclear Science v.43, No.3 (1996).

4. J.Badier et al, Nucl.Instrum.Meth. A348 74 (1994).

5. LHCb Calorimeters, Technical Design Report, CERN/LHCC/2000-0036.

6. LHCb Reoptimized Detector, Design and Performance, Technical Design Report, CERN/LHCC/2003-030.

7. LHCb Trigger System, Technical Design Report, CERN/LHCC/2003-031.

8. P.Aspell et al, Nucl. Instrum. Meth. A376 17 (1996); CMS TN/94-311 (1994) 